\title{
IFITM6 expression is increased in macrophages of tumor-bearing mice
}

\author{
JEONG HYE HAN, SUNYI LEE, YUN SUN PARK, JEONG SU PARK, KUN-YONG KIM, \\ JONG SEOK LIM, KI SOOK OH and YOUNG YANG
}

Department of Biological Science, Sookmyung Women's University, Seoul 140-742, Republic of Korea

Received August 31, 2010; Accepted October 13, 2010

DOI: $10.3892 /$ or.2010.1092

\begin{abstract}
The family of interferon-induced transmembrane protein (IFITM) genes consists of IFITM1, 2, 3, 5, and 6. They encode cell surface proteins that modulate cell-cell adhesion and cell differentiation. In a previous study, we showed that IFITM1 is involved in the immune escape and metastasis of gastric cancer cells. In this study, we determined the difference in expression of IFITM family genes in tumorbearing mice. IFITM1 and 6 were found to be significantly increased. IFITM6 gene expression was increased only in the spleen of tumor-bearing mice but not in the bone marrow, lymph node, or thymus. IFITM6 expression was induced in various macrophages, including splenic, thioglycollate-elicited, and bone marrow-derived macrophages, but not in $\mathrm{T}$ cells. Lipopolysaccharides (LPS) also increased IFITM6 expression $24 \mathrm{~h}$ after administration, and Toll-like receptor $1,2,3,4$, and 9 agonists stimulated IFITM6 expression. These findings imply that the increase in IFITM6 expression may be involved in macrophage functions of tumor-bearing mice.
\end{abstract}

\section{Introduction}

Protective immune function is impaired early in cancer development, and this dysfunction progresses to metastatic disease. The nature of and molecular mechanisms underlying immune dysfunction are not clearly defined. Potential mechanisms of impaired immune function in cancer include defects in antigen recognition, costimulation, and removal of the target using natural killer cells activated by interferons (IFNs) (1). Although type I IFN- $\alpha$ and $-\beta$ were originally thought to be mainly antiviral agents, recent studies have shown their importance as immunomodulators (2), whereas the immunomodulatory activity of IFN $-\gamma$ has long been appreciated (3). IFNs released by lymphocytes against viral

Correspondence to: Dr Young Yang, Department of Life Science, Sookmyung Women's University, Seoul 140-742, Republic of Korea E-mail: yyang@sookmyung.ac.kr

Key words: interferon-induced transmembrane protein, macrophages, breast cancer, lipopolysaccharides, Toll-like receptor and parasitic infections activate natural killer cells and macrophages. They increase the recognition of infected cells or tumor cells by up-regulating antigen presentation. Binding of IFNs to their receptors results in phosphorylation of JAK1/2 and TYK2. Activated JAK1/2 and TYK2 activate STAT1/2 through phosphorylation. In turn, IFNs induce the expression of several genes to execute their function (4). Among these genes are the IFITM family of genes. IFITM1, 2 , and 3 have been experimentally identified (5), and bioinformatic analysis has further revealed IFITM5 and IFITM6 (6). The IFITM family proteins encode two putative transmembrane domains. IFITM1, also known as 9-27 and Leu13, was first identified as an IFN-inducible gene $(7,8)$. IFITM1 induces the homotypic adhesion of cells (9), and IFITM1expressing tumor cells show resistance to natural killer cell cytotoxicity and an increase in the invasiveness of gastric cancer cells (10). IFITM1 also inhibits extracellular signalregulated kinase-mediated p53 phosphorylation, which results in the stabilization of p53 that, in turn, results in antiproliferative activity (11). IFITM1, 2, and 3 are differentially expressed during development of primordial germ cells (5). IFITM6 expression appears to be much more restricted to macrophages (12).

Previously, we showed that IFITM1 has an immune escape function in gastric cancer cells. Little is known about the function of IFITM2, 3, 5, and 6 in tumor-bearing mice. In this study, we investigated changes in the expression of IFITM family genes in the spleens of tumor-bearing mice. The expression of IFITM6 was increased. Thus, we focused on whether IFITM6 is involved in tumor immune functions.

\section{Materials and methods}

Reagent. Lipopolysaccharide (Escherichia coli, serotype 055:B5) was purchased from Sigma-Aldrich (St. Louis, MO, USA). The mouse Toll-like receptor (TLR) 1-9 Agonist kit was purchased from Invivogen (San Diego, CA, USA). Murine IFN- $\gamma$ and IL-4 were purchased from Peprotech (Seoul, Korea). Phorbol-12-myristate-13-acetate (PMA) and ionomycin were purchased from Sigma-Aldrich.

Cell cultures. The mammary carcinoma cell line 4T1 derived from Balb/c mice and the murine macrophage cell line RAW 264.7 were cultured in Dulbecco's modified Eagle's medium (DMEM; Welgene, Daegu, Korea) supplemented with 10\% 
fetal bovine serum (Hyclone, South Logan, UT, USA), and the cells were maintained at $37^{\circ} \mathrm{C}$ in a humidified atmosphere of $5 \% \mathrm{CO}_{2}$.

Syngeneic tumor model. Eight-week-old and 12-week-old $\mathrm{Balb} / \mathrm{c}$ mice were purchased from Samtako Biokorea (OsanCity, Korea). All mice were housed in a 12-h lights-on/12-h lights-off cycle with free access to standard rodent food and clean water at animal care facilities in Sookmyung Women's University. Cultured 4T1 cells were harvested with $0.05 \%$ trypsin-EDTA (Invitrogen, Carlsbad, CA), washed with 1X phosphate-buffered saline (PBS), and resuspended at a concentration of $1 \times 10^{6}$ cells $/ \mathrm{ml}$ in PBS. A total of $200 \mu 14 \mathrm{~T} 1$ cells were administered to the 12 -week-old Balb/c mice by subcutaneous injection. On day 15 after tumor inoculation, all mice were sacrificed, and spleens, thymuses, tumors, lymph nodes, and bone marrows were collected. All animal experiments were performed as approved by the Ethics committee of our university.

Isolation of macrophages from various tissues in Balb/c mice. Thioglycollate (TG) medium (Difco Laboratories Inc., MI, USA) was prepared according to a previous study (15). The TG medium was intraperitoneally administered, and thioglycollateelicited macrophages (TG-macrophages) were obtained from $\mathrm{Balb} / \mathrm{c}$ mice using peritoneal lavage four days after the injection of TG (15). The harvested cells were washed and incubated in DMEM supplemented with $10 \%$ fetal bovine serum (Hyclone), penicillin, and streptomycin.

Bone marrow cells were isolated from Balb/c mouse tibias and femurs of the hind legs using DMEM containing $10 \%$ fetal bovine serum (Hyclone) and then cultured in DMEM supplemented with $10 \%$ L929-conditioned medium (LCM) as a macrophage colony-stimulating factor. After one day, the cells were washed three times with PBS to remove nonadherent cells and then incubated in DMEM with LCM to obtain bone marrow-derived macrophages for three more days. Then, the medium was replaced with fresh DMEM with $10 \%$ FBS without LCM, and the cells were incubated for $24 \mathrm{~h}$.

Splenocytes were obtained from spleens of Balb/c mice. Spleens were ground up using rubber of $1 \mathrm{ml}$ syringe, and the resulting cells were passed through a cell strainer (pore diameter $70 \mu \mathrm{m}$; BD Falcon, MA, USA) to remove debris. The cells were then incubated in $2 \mathrm{ml}$ of RBC lysis buffer for $2 \mathrm{~min}$ to remove red blood cells (Sigma-Aldrich). The cells were washed twice with PBS and then incubated in RPMI-1640 medium supplemented with $10 \%$ FBS, penicillin, and streptomycin for $3 \mathrm{~h}$ to allow adherence.

Treatment with LPS and Toll-like receptor agonists in vivo and in vitro. Twelve-week-old Balb/c mice were randomly divided into three groups. The mice received an intraperitoneal injection of either distilled water as a control or LPS $(5 \mathrm{mg} / \mathrm{kg})$ for either 3 or $24 \mathrm{~h}$. Then, all mice were sacrificed and spleens were isolated. The macrophages were treated with LPS $(0.1 \mu \mathrm{g} / \mathrm{ml})$ for either 3 or $24 \mathrm{~h}$.

Co-culture. For direct contact co-culture experiments, $2 \times 10^{7}$ splenocytes obtained from 12-week-old Balb/c mice were incubated for $24 \mathrm{~h}$ with $1 \times 10^{6} 4 \mathrm{~T} 1$ breast cancer cells prepared
1 day previously. For the non-contact co-culture experiments, a plate of six $3.0-\mu \mathrm{m}$ pore size transwells (Corning, NY, USA) was used. The thioglycollate-elicited macrophages were cultured in the transwell chamber, and the 4T1 cells were cultured in the bottom of the plate.

$R N A$ extraction and reverse transcription- $P C R$. The total cellular RNA from tissues and cells was extracted using the RNAiso plus kit (Takara, Shiga, Japan). A total of $3 \mu \mathrm{g}$ RNA were transcribed with $0.5 \mu \mathrm{g}$ of random primer using reverse transcriptase (Fermentas, Ontario, Canada) at $42^{\circ} \mathrm{C}$ for $1 \mathrm{~h}$. The following primer sequences used for the amplification: IFITM1, forward primer (5'-GGAGCAGCAAGAGGTG GTTG-3') and reverse primer (5'-GATGTTCAGGCACTTG GCGG-3'); IFITM2, forward primer (5'-TCTTGTCCACC AATGCCGGG-3') and reverse primer (5'-AACCACATC GCCCACCATCT-3'); IFITM3, forward primer (5'-CAA GCCTTCATCACCGCTGC-3') and reverse primer (5'-GGG CTCCAGTCACATCACCC-3'); IFITM5, forward primer (5'-TCCATCATCCCGCAAGGCTG-3') and reverse primer (5'-ATGGGGGCACCAATGTCCAC-3'); IFITM6, forward primer (5'-GGTTAAGAGGGATCC-3') and reverse primer (5'-CTTTGACAGTGCATG-3'); IL-1ß, forward primer (5'-ACAGATGAAGTGCTCCTTCCA-3') and reverse primer (5'-GTCGGAGATTCGTAGCTGGAT-3'); IL-2, forward primer (5'-CTGCCACCTAAGTGTGGGCT-3') and reverse primer (5'-GTACATGCCTGCAGGACTTGAGG-3'); IL-6, forward primer (5'-GAAATGAGAAAAGAGTTGTGC-3') and reverse primer (5'-ATTGGAAATTGGGGTAGGAAG-3'); IFN- $\gamma$, forward primer (5'-CTTATTGGGACAATCTC-3') and reverse primer (5'-GCCAGATTATCTCTTTCTAC-3'); TNF- $\alpha$, forward primer (5'-ATGAGCACAGAAAGCATGA-3') and reverse primer (5'-TACAGGCTTGTCACTCGAATT-3'); B-actin, forward primer (5'-GTGGGGCGCCCCAGGCA CCA-3') and reverse primer (5'-CTCCTTAATGTCACGCAC GAT-3'). PCR was performed with 30 cycles of amplification. Each amplification cycle consisted of $30 \mathrm{sec}$ of denaturation at $94^{\circ} \mathrm{C}, 30 \mathrm{sec}$ of annealing at $55^{\circ} \mathrm{C}$, and $30 \mathrm{sec}$ of extension at $72^{\circ} \mathrm{C}$; and PCR was performed on a PTC-100 from MJ Research Inc. (Waltham, MA, USA). Amplification of $B$-actin was used as the control. PCR products were separated on a $1 \%$ agarose gel and photographed.

\section{Results}

Expression pattern of the IFITM family in lymphoid organs of tumor-bearing mice. To determine the function of the IFITM family in tumors, the 4T1 mouse mammary tumor cell line was inoculated into syngeneic Balb/c mice, and spleens were prepared two weeks after $4 \mathrm{~T} 1$ injection. The size of the spleen was greatly increased in tumor-bearing mice (Fig. 1A). The expression pattern of the IFITM family in spleens was determined using RT-PCR analysis. IFITM1 and IFITM6 expression was increased compared to control mice, and the most strongly increased gene was IFITM6 (Fig. 1B). Thus, we focused on the role of IFITM6 expression in tumor-bearing mice.

Next, we examined expression patterns of IFITM6 in various lymphoid organs, including the bone marrow, lymph node, and thymus. Bone marrow showed a high expression of IFITM6 in normal mice, and expression was weakly 
A

Tumor-bearing mice

Spleen

$\mathrm{N}$

$T$

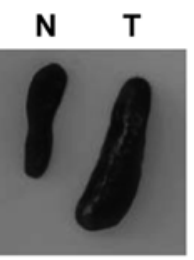

C
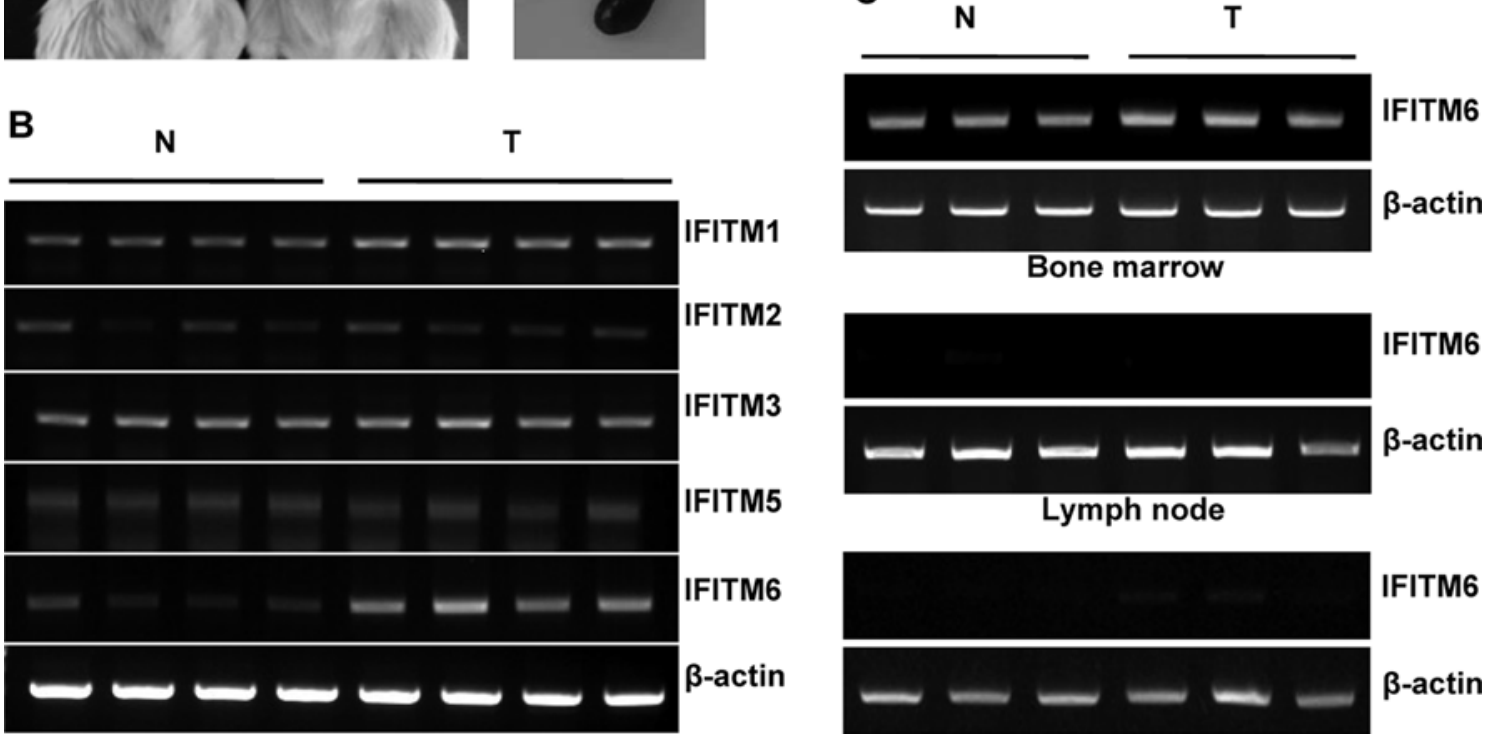

IFITM1

B $\quad \mathrm{N}$

T
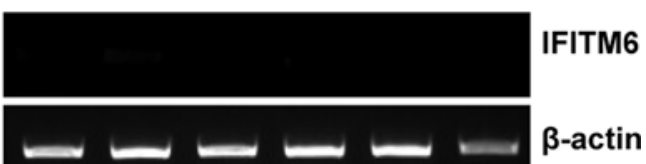

$\beta$-actin

Lymph node

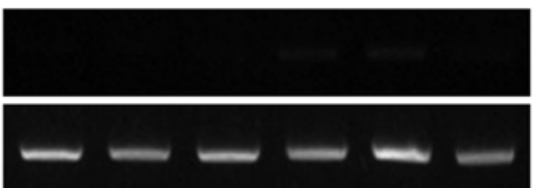

IFITM6

Thymus

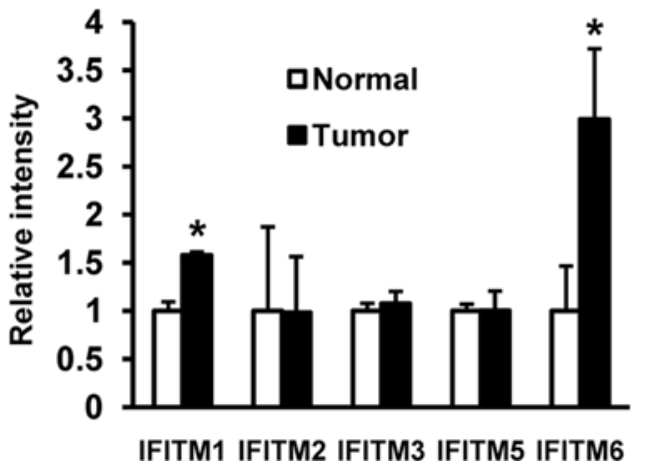

Figure 1. Expression pattern of IFITM family genes in tumor-bearing mice. (A) Twelve-week-old Balb/c mice were injected with 4T1 tumor cells, tumors were grown and spleens were photographed two weeks after the injection. (B) Total RNA was isolated from splenocytes taken from normal and tumorbearing mice. The expression level of the IFITM 1, 2, 3, 5, and 6 genes were determined using RT-PCR analysis. RT-PCR bands were quantitated using Gelquant software (DNR Bio-imaging Systems) and graphed. B-actin was used as a control. Three independent experiments were performed, and data from one representative trial are shown. ${ }^{*} \mathrm{P}<0.05$, control versus tumor. (C) Total RNA was isolated from the bone marrow, lymph node, and thymus taken from normal and tumor-bearing mice. The expression level of the IFITM 6 gene was determined using RT-PCR analysis. Two independent experiments were performed, and data from one representative trial are shown. N, normal mice; $\mathrm{T}$, tumor-bearing mice.

increased in tumor-bearing mice. The lymph node and thymus showed a small amount of IFITM6 (Fig. 1C).

IFITM6 is expressed in lymphoid organ-derived macrophages. Macrophages and cytolytic T lymphocytes are major immune defense cells against tumor cells. To examine whether IFITM6 is involved in tumor defense of the immune system, macrophages were isolated from spleens, peritoneal cavities, and bone marrows of normal and tumor-bearing mice. IFITM6 expression was then determined using RT-PCR analysis. All macrophages derived from the lymphoid organs of tumor-bearing mice showed marked expression of IFITM6 (Fig. 2A). Bone marrow-derived cells from both normal and tumor-bearing mice expressed IFITM6 at high levels (Fig. 1C), but bone marrow-derived macrophages (BMDMs) showed a significant increase in IFITM6 expression only in tumor-bearing mice. To determine whether $\mathrm{T}$ cell activation is responsible for the induction of IFITM6 expression, splenocytes were activated by treatment with PMA and ionomycin. Whereas expression levels of the positive controls IL-2 and IFN $-\gamma$ were increased, IFITM6 expression was not increased (Fig. 2B), indicating that $\mathrm{T}$ cell activation may not be involved in IFITM6 expression, at least in vitro.

IFITM6 expression is increased in spleens of LPS-treated Balb/c mice. To determine whether LPS could induce IFITM6 expression, LPS was administrated to Balb/c mice, and spleens were isolated at the indicated time points. The levels of IFN- $\gamma$ and $\mathrm{TNF}_{-} \alpha$ expression were increased $3 \mathrm{~h}$ after LPS administration, and IFITM6 expression was significantly induced at 
A

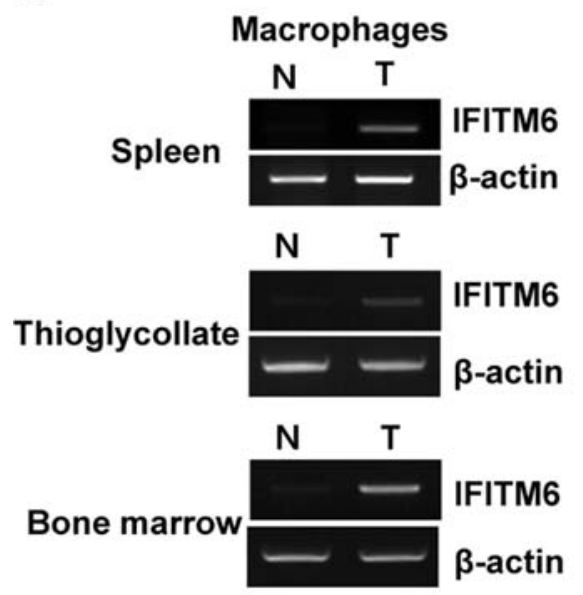

B

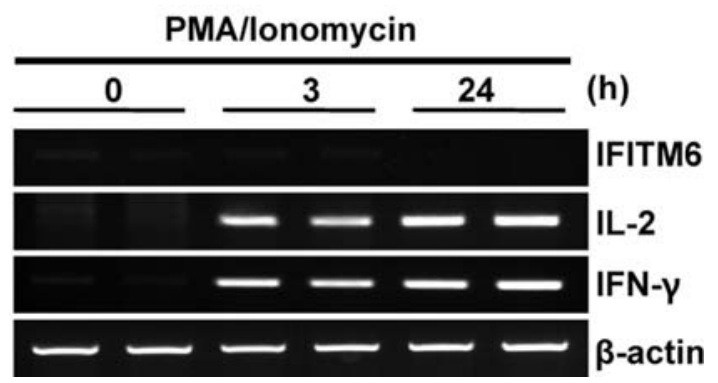

Figure 2. Increase in IFITM6 gene expression in macrophages of tumorbearing mice. (A) Macrophages from various tissues were prepared from normal and tumor-bearing mice, and the total RNA was isolated. The expression level of the IFITM 6 gene was determined using RT-PCR analysis. Three independent experiments were performed, and data from one representative trial are shown. $\mathrm{N}$, normal mice; $\mathrm{T}$, tumor-bearing mice. (B) Normal splenocytes were treated with $1 \mu \mathrm{g} / \mathrm{ml}$ phorbol-12-myristate-13acetate and $5 \mu \mathrm{M}$ ionomycin, and total RNA was isolated 3 and $24 \mathrm{~h}$ after treatment. The expression levels of IL-2, IFN- $\gamma$, and IFITM6 genes were determined using RT-PCR analysis.

24 h (Fig. 3). Next, we screened which Toll-like receptor agonists could induce IFITM6 expression. RAW 264.7 macrophages were treated with various Toll-like receptor agonists. The stimulation of RAW 264.7 cells with agonists for Toll-like receptors 1, 2, 3, 4, and 9 increased IFITM6 expression (Fig. 4). Flagellin from Salmonella typhimurium (TLR5 agonist), a synthetic lipoprotein LP44 (TLR6/2 agonist), and a singlestranded RNA oligonucleotide (TLR7 agonist) failed to stimulate the expression of IFITM6, indicating that many TLR ligands can increase IFITM6 expression. Since the TLR4 agonist LPS induced IFITM6 expression in vivo, RAW 264.7 cells were stimulated with LPS, and total RNA was isolated at the indicated time points. IFITM6 expression was induced at $3 \mathrm{~h}$ and increased to $24 \mathrm{~h}$, which was similar to the induction pattern of IL-6 after LPS stimulation (Fig. 5A). Macrophages can be activated either classically or alternatively. LPS activates macrophages classically, and the activated macrophages secrete pro-inflammatory cytokines. IL-4 stimulates macrophages alternatively, and the activated macrophages secrete anti-inflammatory cytokines. Thus, we determined

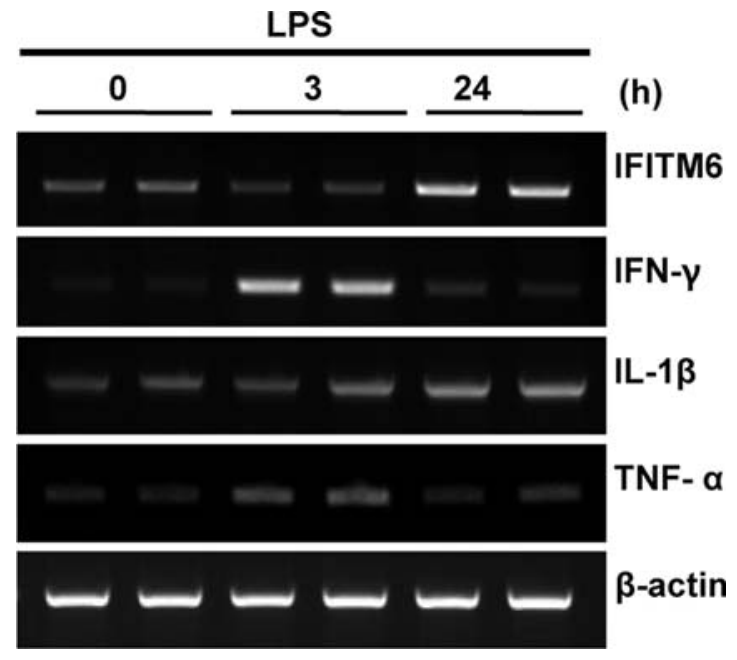

Figure 3. Expression of the IFITM6 gene in spleens of LPS-treated Balb/c mice in vivo. Twelve-week-old Balb/c mice were intraperitoneally administered with $5 \mathrm{mg} / \mathrm{kg}$ LPS, and spleens were removed 3 and $24 \mathrm{~h}$ after the LPS injection. Total RNA was isolated from splenocytes prepared from spleens, and the expression levels of the IFITM6, IFN- $\gamma$, IL-1ß, and TNF- $\alpha$ genes were determined using RT-PCR analysis.

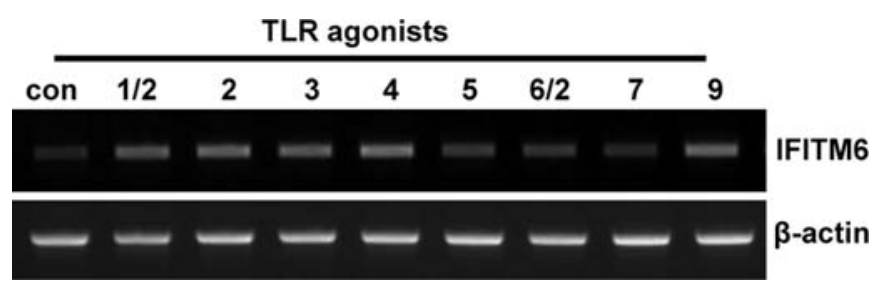

Figure 4. Expression pattern of IFITM6 in TLR 1-9 agonist-treated RAW 264.7 cells. RAW 264.7 cells were treated with TLR agonists for $24 \mathrm{~h}$, and total RNA was isolated. The expression level of IFITM6 in RAW 264.7 cells activated by TLR agonists was analyzed using RT-PCR. TLR 1/2 agonist, Pam3CSK4 (0.1 $\mu \mathrm{g} / \mathrm{ml})$; TLR2 agonist, HKLM $\left(10^{8}\right.$ cells $\left./ \mathrm{ml}\right)$; TLR3 agonist, poly(I:C) LMW (10 $\mu \mathrm{g} / \mathrm{ml})$; TLR4 agonist, LPS-EK $(0.1 \mu \mathrm{g} / \mathrm{ml})$; TLR5 agonist, ST-FLA (50 ng/ml); TLR6/2 agonist, FSL1 (1 $\mu \mathrm{g} / \mathrm{ml})$; TLR7 agonist, ssRNA40 $(0.5 \mu \mathrm{g} / \mathrm{ml})$ and TLR9 agonist, ODN1826 $(5 \mu \mathrm{M})$.

whether IL-4 could stimulate IFITM6 expression. IL-4 reduced the basal level of IFITM6 expression, but it failed to suppress LPS-stimulated IFITM6 expression (Fig. 5B).

Next, we determined whether co-culture of 4T1 tumor cells with splenocytes induced an increase in IFITM6 expression. Splenocytes were prepared from Balb/c mice and mixed with $4 \mathrm{~T} 1$ cells. The unattached splenocytes and attached 4T1 cells were separately collected by pipetting $24 \mathrm{~h}$ after co-culture. Total RNA from both cells was isolated, and IFITM6 expression was examined using RT-PCR. Neither cell line showed IFITM6 expression (Fig. 6A). Macrophages are able to be recruited into tumor tissues. Thus, it is possible for tumor-recruited macrophages to express the IFITM6 gene within tumor tissues even though 4T1 cells do not express the IFITM6 gene. Therefore, IFITM6 expression was determined in four tumor tissues. The IFITM6 gene was weakly detected within tumor tissues (Fig. 6B). 
A

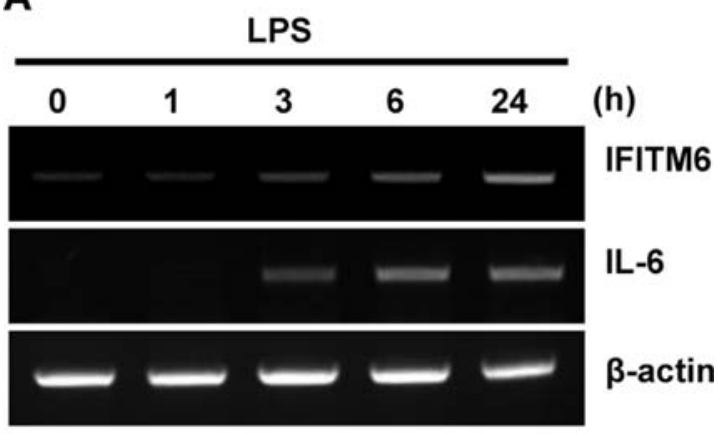

B

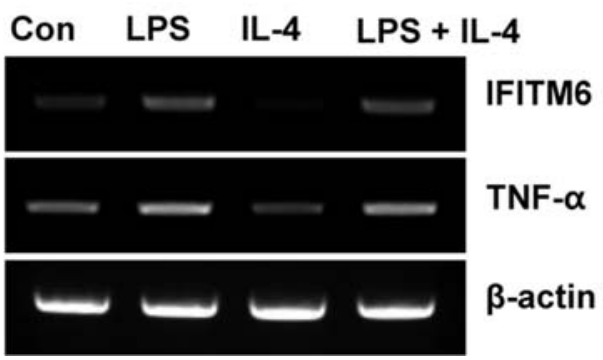

Figure 5. Gene expression pattern of IFITM6 in RAW 264.7 cells treated with LPS $(0.1 \mu \mathrm{g} / \mathrm{ml})$ or IL-4 $(10 \mathrm{ng} / \mathrm{ml})$. (A) RAW 264.7 cells were treated with $0.1 \mu \mathrm{g} / \mathrm{ml}$ LPS, and total RNA was isolated. Expression levels of the IFITM6 and IL-6 genes were determined using RT-PCR analysis. (B) RAW 264.7 cells were treated with $0.1 \mu \mathrm{g} / \mathrm{ml}$ LPS in the presence or absence of $10 \mathrm{ng} / \mathrm{ml} \mathrm{IL-4,} \mathrm{and} \mathrm{total} \mathrm{RNA} \mathrm{was} \mathrm{isolated.} \mathrm{Expression} \mathrm{levels} \mathrm{of} \mathrm{IFITM6}$ and TNF- $\alpha$ genes were determined using RT-PCR analysis.

\section{Discussion}

IFITM1 negatively regulates cell growth, and suppression of IFITM1 blocks the anti-proliferative effect of IFN- $\gamma$. IFITM1 also enhances the transcriptional activity of p53 and stabilizes the $\mathrm{p} 53$ protein (11). IFITM1, therefore, mediates the antiproliferative activity of IFN- $\gamma$ to tumor cells. Increased IFITM1 expression is observed in gastric cancer, and overexpression of IFITM1 shows an increased migration and invasive capacity of gastric cancer cells and resistance to NK cytotoxicity (10). IFITM1, IFITM2, and IFITM3 gene expression is strongly induced by Wnt/B-catenin signaling, and up-regulation of these genes is highly specific to human colorectal carcinogenesis. In a murine model, a strong induction of IFITM1, IFITM2, and IFITM3 gene expression was observed regardless of the tumor stage, but IFITM6 was only induced in adenomas (13). Although the functional significance of IFITM gene induction remains to be elucidated, previous results imply that the increased expression of IFITM1, IFITM2, and IFITM3 genes in tumor cells and tissues may play an important role in tumor development and progression. In this study, we measured IFITM6 expression in 4T1 mammary cancer cells and 4T1derived tumor tissues, but no expression was observed (data not shown), indicating that IFITM6 itself is not associated with a function in mammary tumor cells because IFITM6 is a transmembrane protein.

IFITM1, IFITM2, and IFITM3 are expressed in a wide variety of immune cells, including bone marrow, bone marrow-derived macrophages, and mucosal mast cells. They
A

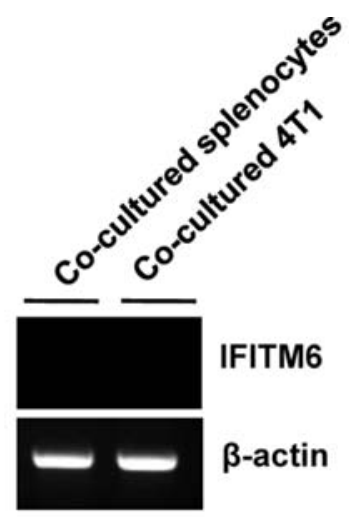

B

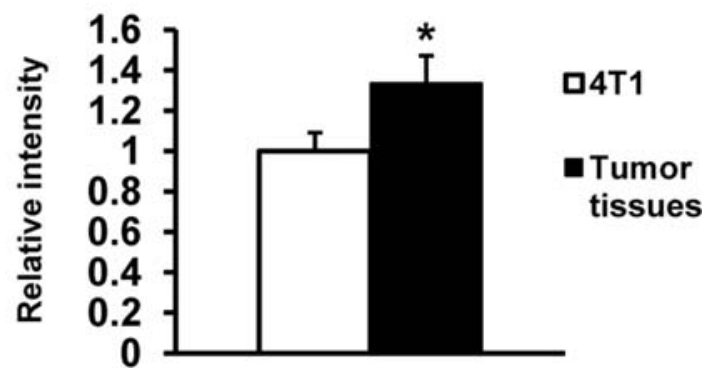

Figure 6. Gene expression pattern of IFITM6 in co-cultured 4T1 cells or splenocytes and 4T1 tumor tissues. (A) A total of $2 \times 10^{7}$ splenocytes obtained from Balb/c mice were incubated with $1 \times 10^{6} 4 \mathrm{~T} 1$ tumor cells for $24 \mathrm{~h}$, and total RNA was isolated from each cell type. The expression level of the IFITM6 gene was determined using RT-PCR analysis. (B) Twelve-week-old $\mathrm{Balb} / \mathrm{c}$ mice were injected with 4T1 tumor cells, and total RNA was isolated from tumor tissues two weeks after 4T1 tumor cell injection. The expression level of the IFITM6 gene was determined using RT-PCR analysis. RT-PCR bands were quantitated using Gelquant software (DNR Bio-Imaging Systems) and graphed. ${ }^{*} \mathrm{P}<0.05$, normal versus tumor tissues.

are also expressed in a variety of immune cell lines, including RAW 264.7, EL4 (T cell line), and 2PK3 (B cell line), but IFITM6 expression is restricted to RAW 264.7 and bone marrow (12). IFITM1 and IFITM3 are translocated to lipid rafts along with tetraspanin protein CD81, CD19, and CD21 following $B$ cell activation, indicating that IFITM1 and IFITM3 may play a role in B cell activation. IFITM6, however, is not associated with B cell activation (12). Consistent with the previous results, IFITM6 expression was observed mainly in the bone marrow and weakly in RAW 264.7 cells. In this study, we showed that IFITM6 expression was strongly induced in spleens following tumor formation using a syngeneic tumor model. Splenic macrophages were responsible for tumor-induced IFITM6 expression in the spleens of tumorbearing mice. The expression level of IFITM6 was highest in the bone marrow of normal mice, but bone marrow-derived macrophages showed a low level of expression in normal mice (Fig. 2A). However, when macrophages were derived from bone marrow isolated from tumor-bearing mice, a high level of IFITM6 expression was observed similar to splenic and thioglycollate-elicited macrophages. Various TLR agonists were also able to stimulate IFITM6 expression in the RAW 264.7 macrophage cell line. These results imply that IFITM6 may play a role in macrophages. It remains to be elucidated whether the increase in IFITM6 in the macrophages of 
tumor-bearing mice is for anti-tumor activity or a result of tumor-induced immune suppression.

It is well understood that the majority of malignant tumors contain numerous macrophages as a major component of leukocyte infiltrate. These macrophages are referred to as tumor-associated macrophages (TAMs), and most are derived from peripheral blood monocytes recruited into the tumor mass. Upon activation, TAMs release various inflammatory cytokines and proteolytic enzymes, which play a critical role in tumor progression (14). Our data also showed that tumor tissues expressed IFITM6 (Fig. 6B). Since 4T1 cells did not express the IFITM6 gene and macrophages were able to express it, we think that tumor-infiltrating macrophages are responsible for IFITM6 expression within tumor tissues.

In conclusion, expression of the IFITM6 gene was most strongly induced in the spleen of tumor-bearing mice, and the macrophages were the main cells expressing the IFITM6 gene. In general, because tumor-activated macrophages promote tumor progression, macrophages expressing IFITM6 may be involved in tumor-promoting functions, and further study of the IFITM6 gene is needed to elucidate these functions.

\section{Acknowledgements}

This study was supported by a grant of the Korea Healthcare Technology R\&D Project, Ministry for Health, Welfare and Family Affairs, Republic of Korea (A08-4673-AA202308N1-00010A).

\section{References}

1. Reiter Z: Interferon - a major regulator of natural killer cellmediated cytotoxicity. J Interferon Res 13: 247-257, 1993.

2. Decker T, Muller M and Stockinger S: The yin and yang of type I interferon activity in bacterial infection. Nat Rev Immunol 5: 675-687, 2005.

3. Berenson LS, Ota $\mathrm{N}$ and Murphy KM: Issues in T-helper 1 development - resolved and unresolved. Immunol Rev 202: 157-174, 2004

4. Dunn GP, Koebel CM and Schreiber RD: Interferons, immunity and cancer immunoediting. Nat Rev Immunol 6: 836-848, 2006
5. Tanaka SS and Matsui Y: Developmentally regulated expression of mil-1 and mil-2, mouse interferon-induced transmembrane protein like genes, during formation and differentiation of primordial germ cells. Mech Dev 119 (Suppl 1): 261-267, 2002.

6. Tanaka SS, Nagamatsu G, Tokitake Y, Kasa M, Tam PP and Matsui Y: Regulation of expression of mouse interferoninduced transmembrane protein like gene-3, Ifitm3 (mil-1, fragilis), in germ cells. Dev Dyn 230: 651-659, 2004.

7. Lewin AR, Reid LE, McMahon M, Stark GR and Kerr IM: Molecular analysis of a human interferon-inducible gene family. Eur J Biochem 199: 417-423, 1991.

8. Ackrill AM, Reid LE, Gilbert CS, et al: Differential response of the human 6-16 and 9-27 genes to alpha and gamma interferons. Nucleic Acids Res 19: 591-598, 1991.

9. Evans SS, Collea RP, Leasure JA and Lee DB: IFN-alpha induces homotypic adhesion and Leu-13 expression in human B lymphoid cells. J Immunol 150: 736-747, 1993.

10. Yang Y, Lee JH, Kim KY, et al: The interferon-inducible 9-27 gene modulates the susceptibility to natural killer cells and the invasiveness of gastric cancer cells. Cancer Lett 221: 191-200, 2005.

11. Yang $\mathrm{G}, \mathrm{Xu} \mathrm{Y}$, Chen $\mathrm{X}$ and Hu G: IFITM1 plays an essential role in the antiproliferative action of interferon-gamma. Oncogene 26: 594-603, 2007.

12. Smith RA, Young J, Weis JJ and Weis JH: Expression of the mouse fragilis gene products in immune cells and association with receptor signaling complexes. Genes Immun 7: 113-121, 2006.

13. Andreu P, Colnot S, Godard C, et al: Identification of the IFITM family as a new molecular marker in human colorectal tumors. Cancer Res 66: 1949-1955, 2006.

14. Varney ML, Johansson SL and Singh RK: Tumour-associated macrophage infiltration, neovascularization and aggressiveness in malignant melanoma: role of monocyte chemotactic protein-1 and vascular endothelial growth factor-A. Melanoma Res 15: 417-425, 2005.

15. Li YM, Baviello G, Vlassara H and Mitsuhashi T: Glycation products in aged thioglycollate medium enhance the elicitation of peritoneal macrophages. J Immunol Methods 201: 183-188, 1997. 